\title{
La guerre du chalut
}

Résister au changement technique dans le monde de la pêche (Dieppe, 1818)

War on trawling. Resisting technological change in the fishing industry

\section{Romain Grancher}

\section{(2) OpenEdition \\ 1 Journals}

Édition électronique

URL : https://journals.openedition.org/artefact/9868

DOI : 10.4000/artefact.9868

ISSN : 2606-9245

Éditeur:

Association Artefact. Techniques histoire et sciences humaines, Presses universitaires du Midi

\section{Édition imprimée}

Date de publication : 7 octobre 2021

Pagination : 89-107

ISBN : 978-2-8107-0755-3

ISSN : 2273-0753

\section{Référence électronique}

Romain Grancher, «La guerre du chalut », Artefact [En ligne], 14 | 2021, mis en ligne le 07 octobre 2021, consulté le 08 octobre 2021. URL : http://journals.openedition.org/artefact/9868 ; DOI : https:// doi.org/10.4000/artefact.9868

\section{cc) (†) $\odot$}

Artefact, Techniques, histoire et sciences humaines est mise à disposition selon les termes de la Licence Creative Commons Attribution - Pas d'Utilisation Commerciale - Pas de Modification 4.0 International. 


\section{La guerre du chalut}

\section{Résister au changement technique dans le monde de la pêche (Dieppe, 1818) \\ Romain Grancher}

\section{Résumé}

Cet article part du récit d'une émeute de gens de mer survenue dans le port de Dieppe au mois novembre 1818. Dirigée contre des pêcheurs étrangers à la communauté, cette émeute est envisagée ici comme la trace d'un conflit d'usage de longue durée lié à l'essor du chalut, un filet traînant auquel certains acteurs attribuent la destruction des ressources halieutiques. L'analyse de cet épisode de violence permet ainsi d'enrichir la compréhension des résistances au changement technique en proposant une réflexion à partir du monde de la pêche, à rebours d'une historiographie qui s'est surtout intéressée aux machines industrielles (et agricoles, dans une moindre mesure).

\section{Mots-clés}

pêche, chalut, changement technique, violence, Manche, $x \mid x^{e}$ siècle

99 Romain Grancher, « La guerre du chalut. Résister au changement technique dans le monde de la pêche (Dieppe, 1818) », Artefact, 14, 2021, p. 89-107. 


\section{War on trawling \\ Resisting technological change in the fishing industry}

\section{Abstract}

This article describes the course of a riot that took place in the port of Dieppe in November 1818. Directed against fishermen from outside the community, this riot is the trace of a long-lasting conflict of use linked to the development of the trawl net, which was considered to be destructive of fishery resources. The analysis of this episode of violence provides new elements for understanding the history of resistance to technical change from the world of fishing, in contrast to a historiography that has mainly focused on industrial (and to a lesser extent agricultural) machines.

\section{Keywords}

fishing, trawling, technical change, violence, English Channel, $19^{\text {th }}$ century

la fin du mois de novembre 1818, un mouvement de protesta-
tion marqué par des violences survient dans le port de pêche de
Dieppe (Fig. II, cahier couleur), l'un des principaux lieux d'approvisionnement de Paris et des provinces septentrionales de la France en produits de la mer ${ }^{1}$. Déclenché par des femmes de pêcheurs habitant le faubourg maritime du Pollet, ce mouvement qualifié d' " émeute " par les autorités maritimes est dirigé contre des pêcheurs originaires de Honfleur, en relâche dans le port pour y vendre leur poisson, ainsi qu'ils ont pris l'habitude de le faire quelques années plus tôt, lorsqu'ils ont commencé à venir chaluter sur les riches fonds situés dans les parages de Dieppe ${ }^{2}$. Loin d'être isolé ou spontané, cet épisode de violence constitue l'acmé d'une «sorte

1. Abad, 2002, p. 397-456; Mollat (dir.), 1987, p. 158.

2. Le Pollet est l'un des quartiers maritimes de Dieppe, dans lequel habitent principalement des pêcheurs aux cordes vivant des " petites pêches " côtières, par opposition aux " grandes pêches " du hareng et du maquereau pratiquées par les autres pêcheurs de Dieppe. Sur les quartiers maritimes en général et celui du Pollet en particulier, voir Cabantous, 1995, p. 95-113. 
de guerre " larvée entre ces deux communautés " de la côte du Nord » et de « de la côte du Sud» : celle-ci se déroule dans les premières années de la Restauration et a pour enjeu le chalut, un filet traînant que les équipages du Calvados emploient tout au long de l'année sur les côtes de Haute et Basse-Normandie pour approvisionner les poissonniers et les mareyeurs en marée fraîche ${ }^{3}$.

Soutenus par les représentants du négoce de la place, et en particulier par leurs armateurs, les pêcheurs de Dieppe réclament dans les années 1810 un encadrement plus strict de cet engin (voire, pour certains, son interdiction pure et simple) au motif qu'il serait responsable de ce qu'ils appellent « la dépopulation des côtes " :

Chargé par les côtés d'armures et par le bas de chaines de fer dont le poids va toujours croissant, [il] racle et laboure tous les fonds sur lesquels il passe, déracine et enlève les herbes servant d'abri et de réduit aux poissons, rompt les lits de leur frai, et écrase par sa pesanteur ou emporte sans profit ceux du premier âge. Nul doute qu'un filet de cette nature ne soit éminemment contraire à la propagation du poisson et destructeur des pêches ${ }^{4}$.

Appuyés par les membres du tribunal de commerce de Honfleur, "les pêcheurs de la côte du Sud » récusent ces allégations et revendiquent même la liberté de pêcher quand bon leur semble avec un modèle de chalut beaucoup plus massif et efficace que celui qui avait été consacré sous l'Ancien Régime par l'ordonnance royale du 31 octobre 17445. Appelée à rendre un arbitrage, l'administration de la Marine ordonne au printemps 1818 la tenue d'une assemblée, puis fait procéder à « des expériences propres à éclairer sur les effets du chalut " avant de rendre, sur la base de ces " épreuves ", une nouvelle ordonnance datée du 13 mai 1818 : rappelant le principe de la liberté de la pêche anciennement consacré par l'ordonnance de la marine

3. Vincennes, Service historique de la défense, (ci-après SHDV), Mar., CC 5 628-629, Rapport sur le filet de pêche dit rets traversier ou chalut, 4 mai 1818, p. 1. J'ai déjà abordé ce conflit, d'un autre point de vue et à partir d'autres sources, dans Grancher, 2015.

4. SHDV, Mar., CC 578 , Mémoire contre l'emploi abusif du filet appelé communément chausse, 17 mars 1818 , p. 3-5.

5. Ordonnance du roi, qui rétablit la pêche avec le filet nommé ret traversier ou chalut, depuis le premier septembre jusqu'au dernier avril de chaque année, 31 octobre 1744, reproduite dans Valin, 1760 , tome II, p. 660. 
d'août 1681, celle-ci vient donner une valeur légale aux usages adoptés de fait par les chalutiers de Basse-Normandie depuis plusieurs décennies ${ }^{6}$.

Cet élément de contexte a toute son importance si l'on cherche à comprendre les « logiques de la foule » qui investit les quais de Dieppe dans les derniers jours de novembre 1818 pour empêcher ces chalutiers de débarquer leur poisson ${ }^{7}$. Notons, cela dit, que ce cas n'a rien d'exceptionnel en soi dans la mesure où les violences commises par la population dieppoise à l'encontre de ces pêcheurs " étrangers » sont tout à fait caractéristiques des réactions suscitées au cours des siècles par la lente diffusion du chalut ${ }^{8}$. De ce point de vue, son analyse vaut d'abord en ce qu'elle permet d'enrichir la compréhension des résistances au changement technique dans le monde de la pêche 9 . Très largement négligées par une historiographie restée focalisée sur le "bris de machines » industrielles (et agricoles, dans une moindre mesure), celles-ci présentent pourtant le grand intérêt d'appeler une lecture indissociablement économique, sociale et environnementale des conflits qui se nouent autour des techniques et des mondes qu'elles façonnent ${ }^{10}$.

\section{Une émeute contre le chalut (Dieppe, 28 novembre 1818)}

Repartons des faits, qu'il est possible d'établir précisément grâce à deux sources principales issues des archives de la Marine : d'une part, la correspondance échangée entre le commissaire général du sous-arrondissement maritime Chabanon et l'écrivain des classes de Dieppe Turbest qui, en l'absence du commissaire en charge du quartier, assure alors par intérim

6. SHDV, Mar., CC $528-629$, Rapport sur le filet de pêche dit rets traversier ou chalut, 4 mai 1818, p. 26-28. Il s'agit de l'ordonnance du roi sur l'emploi des filets de pêche dits rets-traversiers ou chalut, et petit chalut à la chevrette, 13 mai 1818 .

7. Farge et Revel, 1988.

8. Les premiers conflits documentés autour du chalut remontent au XIV siècle d'après Jones, 2018. Parmi une bibliographie abondante, voir également Gordon, 1951 ; Uerteaga, 1987, p. 89-103 ; Robinson, 1996, p 14-22 ; Armiero, 1998, p. 193-194 ; Roberts, 2007, p. 130-144 ; Faget, 2011, p. 201-224.

9. Pour une première approche de la question : McCay, 1987, p. 202-208 ; Gersuny et Poggie, 1974. Des travaux plus spécifiques ont été consacrés par exemple à la résistance au développement de la motorisation des embarcations ou à l'introduction du scaphandre (pour la pêche des éponges) : voir respectivement Subramanian, 2003 ; Fourt, Faget et Pérez, 2020.

10. Hobsbawm et Rudé, 1973 ; Thompson, 1988 [1963] ; Bourdeau, Jarrige et Vincent, 2006 ; Jarrige, 2009. 
l'administration de l'inscription maritime et la police des gens de mer ; d'autre part, le long rapport d'enquête rédigé par le commissaire De Raffin, aussitôt dépêché sur les lieux pour rétablir l'ordre et découvrir les individus responsables des violences perpétrées contre les pêcheurs " de la côte du Sud ${ }^{11} »$.

Tout débute, semble-t-il, le 27 novembre au matin, lorsqu'un " attroupement " se forme à proximité de la poissonnerie. À cette occasion, les chalutiers sont désignés publiquement comme les responsables des « mauvais résultats de la pêche » et certaines femmes de maîtres de bateau, parmi «les plus influentes " du Pollet, suggèrent alors aux individus présents de "se réunir pour chasser ces étrangers ${ }^{12}$ ». Quelques temps après, plusieurs équipages de Honfleur venus débarquer leur poisson la veille au soir sont attaqués à coups de pierres alors qu'ils empruntent le chenal pour reprendre la mer. Dans sa déclaration, le maître Goulay dépose qu' " au moment où il sortait du port pour aller faire sa pêche dans les eaux du quartier de Dieppe, des femmes $\&$ des enfants réunis sur la jettée du Pollet, excités par des marins \& d'autres individus qui se tenaient un peu à l'écart, lui ont lancé des pierres, depuis l'épi en bois jusqu'au bout de la jettée, au point qu'il en a ramassé dans son embarcation plus de $150^{13}$ ». D'autres équipages de Honfleur connaissent le même sort avant que la foule ne quitte les quais pour se rendre en cortège chez différents " Messieurs de la ville ", à savoir le maire, le procureur du roi auprès du tribunal de première instance et le président de la chambre de commerce, qui décide de convoquer les maîtres de bateau pour évoquer la question du chalut et entendre leurs doléances. Au vu des témoignages qu'il a pu recueillir, il ne fait aucun doute pour le

11. L'ensemble de la documentation relative à cette émeute (correspondance, rapports, interrogatoires des suspects et dépositions des plaignants) est conservé à Rouen, Archives départementales de la Seine Maritime (ci-après ADSM), 4/M/185. Mis en place à partir de 1665, le système des classes contraint les gens de mer à servir périodiquement dans la marine de guerre en échange d'un monopole sur les activités maritimes. Ce système est réformé par la loi du 3 brumaire an IV et prend alors le nom d'inscription maritime. Au début du XIX ${ }^{e}$ siècle, les pêcheurs sont placés sous l'autorité d'un commissaire qui tient les registres-matricules des marins de son quartier et veille au bon ordre des levées. À ce sujet, voir Captier, 1907 ; Acerra et Zysberg, 1997.

12. ADSM, 4/M/185, Lettre de l'écrivain des classes de Dieppe au commissaire général de la Marine, 28 novembre 1818, 9 heures du matin ; ADSM, 4/M/185, Rapport du commissaire de la Marine en mission, 3 décembre 1818.

13. ADSM, 4/M/185, Registre des rapports et déclarations du bureau de la Marine, 29 novembre 1818. 
commissaire en mission De Raffin que ces entrevues du 27 novembre sont à l'origine des scènes de violence survenues le lendemain.

Le bruit accrédité dans la soirée chez les Polletais fut que ces Messieurs avoient dit qu'il falloit que les femmes et les enfants se portassent contre les barques étrangères [...]. C'est de cet instant seulement que l'insurrection du lendemain fut résolue, parce qu'au désir de chasser des concurrents trop redoutables, se joignit la conviction que les moyens violents que l'on alloit employer à cet effet étoient conseillés par les magistrats et les notables de la ville ; ainsi le premier brandon de la discorde, jetté par les propos raconté le matin par les poissonnières, a été attisé par les diverses démarches faites chez trois magistrats \& surtout par la convocation à la chambre de commerce ${ }^{14}$.

Le lendemain dès l'aube, des " conciliabules " se tiennent dans le port ; deux pêcheurs du Pollet, François Granché et Casimir Casselot (considérés comme les principaux " fauteurs de l'insurrection " par De Raffin) décident alors de se porter chez la Cavette, la crieuse publique, afin qu'elle réveille le faubourg " en criant que tout le monde se rend[e] à la poissonnerie $^{15}$ ». Quelques heures plus tard, on vient effectivement avertir l'écrivain des classes Turbest que « la population entière du Pollet [est] en insurrection, que les femmes, les enfants et des individus de toutes professions se port[ent] sur les quais en jettant des cris affreux " pour s'opposer à l'accostage des chalutiers de Honfleur ${ }^{16}$. S'ensuivent des altercations qui durent toute la matinée du 28 novembre et dont on peut se faire une idée à travers la déposition d'un plaignant, le maître Bastien Voisambert :

À peine étions-nous amarrés que mon équipage et moi entendîmes un bruit confus qui s'élevait de toutes parts dans le Pollet. Alors Godefroy, marin de Dieppe qui était à notre bord nous dit qu'il s'appercevait d'une rumeur populaire dont nous étions l'objet et nous invita à ne pas débarquer notre poisson. À l'instant même, la populace accourut de tous côté [...]. Une foule de femmes, d'enfants et d'hommes, dont le nombre nous parut incalculable,

14. ADSM, 4/M/185, Rapport du commissaire de la Marine en mission, 3 décembre 1818.

15. ADSM, 4/M/185, Rapport du commissaire de la Marine en mission, 3 décembre 1818.

16. ADSM, 4/M/185, Lettre l'écrivain des classes de Dieppe au commissaire général de la Marine, 28 novembre 1818, 9 heures du matin. 
nous accable de menaces, en disant que nous étions des brigands, que nous venions manger leur pain, et mille autres injures de ce genre. Cette populace exaspérée nous défend de débarquer notre poisson, l'effervescence du peuple était au comble. Une grande quantité d'homme présens nous criaient : nous vous prévenons en amis de vous en retourner sans débarquer votre poisson, sinon vous êtes assassinés.

Voisambert aurait même vu parmi cette foule " deux ou trois femmes qui tenaient à la main un couteau fermé et qui s'écriaient avec fureur : tu ne vendras pas ton poisson ${ }^{17}$ ". Certains maîtres de Honfleur tentent néanmoins de mettre pied à terre, prétextant la nécessité de s'approvisionner en vivres avant de reprendre la mer. Plusieurs sont pris à parti par la foule; d'autres sont arrêtés dans le port avant même d'avoir pu atteindre les quais par des pêcheurs du Pollet qui les abordent et les contraignent à repartir ${ }^{18}$. Seule l'intervention de la gendarmerie et des magistrats locaux vient dénouer la situation en fin de matinée. Trois arrestations ont lieu à la suite d'actes de rébellion; puis, tandis qu'une partie des forces de l'ordre reste sur les quais pour surveiller les chalutiers qui ont enfin pu accoster, le reste escorte les pêcheurs de Honfleur jusqu'à la poissonnerie, où la marée fraîche se vend difficilement car les mareyeurs ont "reçu défense d'acheter ${ }^{19}$ ". Rendu chez son " hôtesse ", la cabaretière chez laquelle il a l'habitude de loger lorsqu'il est à Dieppe, Bastien Voisambert apprend par exemple que le mari de cette dernière a été " menacé d'être jetté à la mer s'il continuait de vendre le poisson des pêcheurs de Honfleur ${ }^{20}$ ». La foule finit par se disperser au début de l'après-midi alors que " les esprits fermentent encore " selon Turbest, qui signale à sa hiérarchie que "des menaces ont été proférées par le peuple lorsqu'il a été obligé d'évacuer les quais ». De fait, dans l'après-midi, le maître Jacques Ville s'en prend violemment à Chauvin, « matelot de Dieppe, embarqué momentanément sur

17. Honfleur, Archives municipales (ci-après AMH), F 228, Procès-verbal des troubles qui ont eu lieu à Dieppe, $1^{\text {er }}$ décembre 1818 .

18. ADSM, 4/M/185, Procès-verbal des interrogatoires des 30 novembre, $1^{\text {er }}$ et 2 décembre 1818. 19. ADSM, 4/M/185, Lettre de l'écrivain des classes de Dieppe au commissaire général de la Marine, 28 novembre 1818, 2 heures de l'après-midi.

20. AMH, F 228, Procès-verbal des troubles qui ont eu lieu à Dieppe, $1^{\text {er }}$ décembre 1818. 
une barque de Honfleur ", avant d'être arrêté alors qu'il continue à " tenir sur le port des propos tendant à la révolte ${ }^{21}$ ».

Finalement, un nouvel attroupement se forme dans la soirée sur les jetées du Pollet, à la faveur de l'obscurité. Comme la veille, des grêles de pierres pleuvent sur les équipages de Honfleur alors qu'ils quittent le port où ils sont entrés si difficilement le matin même. Parmi tous les épisodes qui marquent les deux journées des 27 et 28 novembre, celui-ci se distingue par une violence accrue. Pierre Bréval, maître d'une barque de Honfleur, déclarera plus tard que des pierres "de toute grosseur tombaient à son bord " et qu'il a été atteint au bras. André Duchemin, déjà inquiété le 27 au matin, prétend quant à lui avoir " échappé à une mort certaine " en se mettant à "l'abri de sa voile ". Enfin, le fils du maître Goulay, embarqué comme mousse à bord de la barque de son père, est "grièvement blessé " par une pierre reçue à la tête ${ }^{22}$.

Qualifié dans les sources d' "insurrection ", d' « émeute » ou simplement de "rixe ", cet événement suscite une réaction immédiate de la part des autorités en charge du sous-arrondissement maritime, qui dépêchent aussitôt le commissaire de Raffin sur les lieux. Arrivé à Dieppe le 30 novembre au matin, il s'entretient avec les notables locaux, procède à l'interrogatoire de treize individus qui lui ont été désignés comme les principaux acteurs de l'émeute et fait engager des poursuites contre neuf d'entre eux, à savoir sept pêcheurs et deux femmes originaires du Pollet ${ }^{23}$. Les chefs d'accusation retenus contre eux sont les suivants : provocation à la rébellion, rébellion envers la force publique et coups et blessures avec préméditation. Tous sont appelés à comparaître devant le tribunal de première instance en janvier 1819 et se voient alors condamnés à quinze jours d'emprisonnement, ainsi qu'à une amende de cinq francs ${ }^{24}$.

21. ADSM, 4/M/185, Lettre de l'écrivain des classes de Dieppe au sous-préfet de Dieppe, 28 novembre 1818, 2 heures de l'après-midi.

22. ADSM, 4/M/185, Registre des rapports et déclarations du bureau de la Marine, 29 novembre 1818.

23. ADSM, 4/M/185, Lettre du commissaire de la Marine en mission au procureur du roi près le tribunal de première instance de Dieppe, 2 décembre 1818.

24. ADSM, 4/M/185, 3/U/1/671, Jugement rendu le 15/01/1819. 


\section{Défense d'un monde}

Quelle signification attribuer à cet épisode de protestation populaire ? On le sait grâce à une longue tradition d'histoire " d'en bas », les actions de la foule, tout comme les multiples formes de la violence collective, obéissent à des logiques complexes : à ce titre, elles ne sauraient donc être réduites à « de simples réponses à des stimuli économiques ", conformément à une "vision spasmodique » et condescendante que l'on retrouve souvent dans les sources produites d'en haut ${ }^{25}$ - " cette classe-là ne raisonne pas quand elle a faim ${ }^{26}$ " explique ainsi le commissaire général de la Marine dans une lettre adressée à son ministre de tutelle... Certes, la "disette du hareng " qui sévit alors dans la Manche depuis plusieurs saisons consécutives " plonge dans la misère la plus profonde les pêcheurs " et leurs familles ${ }^{27}$. Toutefois, cet événement s'inscrit d'abord dans un contexte de confrontation entre deux mondes que leurs usages et leurs intérêts respectifs semblent opposer de manière irréductible. Envisagées sous cet angle, les violences commises à l'encontre des chalutiers "de la côte du Sud " peuvent être comprises comme un moyen jugé légitime par une partie importante de la communauté (y compris certains notables) pour empêcher la diffusion d'une nouvelle pratique menaçant de déstabiliser l'organisation de cette branche d'activité cruciale pour l'économie locale qu'est la pêche.

25. Thompson, 2015 [1991], p. 251-253.

26. ADSM, 4/M/185, Lettre du commissaire général de la Marine au ministre de la Marine et des Colonies, 2 décembre 1818.

27. ADSM, 4/M/185, Lettre du commissaire général de la Marine au ministre de la Marine et des Colonies, 2 décembre 1818. 


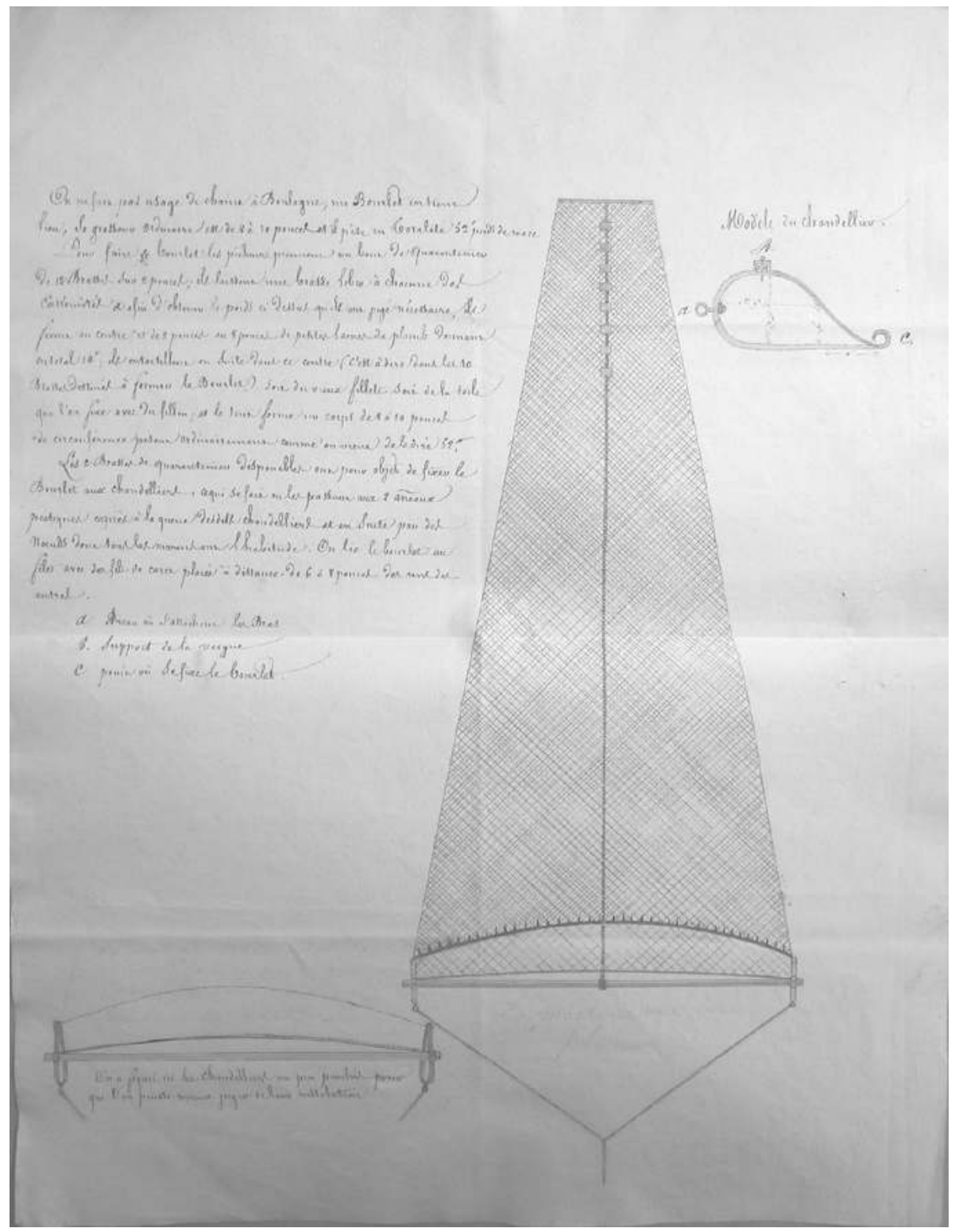

Fig. 1. - Modèle de chalut employé sur les côtes de la Manche dans les années 1820

SHDV, CC5 578, Rapport sur le chalut, 23 avril 1822.

Cliché Romain Grancher 
Le chalut est d'abord considéré comme une technique destructrice (Fig. 1), à laquelle les pêcheurs locaux attribuent la "dépopulation " de leurs côtes et partant les mauvais résultats de leurs pêches, en l'occurrence celle du hareng qui bat normalement son plein à cette saison et celle du "poisson frais ", qui constitue la seule ressource de toute une partie de la population maritime du Pollet dans l'incapacité matérielle d'armer pour la première trop coûteuse. On sait en effet que le hareng " disparaît " presque de la Manche entre 1816 et 1821 , occasionnant une série de saisons catastrophiques qui se répercutent sur toute la population intéressée à cette pêche, depuis les armateurs et les mareyeurs jusqu'aux pêcheurs, en passant par les fabricants de filets ou de barils, les manutentionnaires du port et les ouvriers et ouvrières des ateliers de salaison ${ }^{28}$. Ainsi, il n'est pas surprenant que la foule qui se réunit à plusieurs reprises entre le 27 et le 28 novembre 1818 déplore " les mauvais résultats de la pêche du hareng ${ }^{29}$ ". Mais qu'elle les attribue à l'usage du chalut, voilà qui est plus intrigant, dans la mesure où ce filet traînant n'est pas destiné à la capture de ce poisson pélagique migrateur, mais seulement à celle d'espèces benthiques telles que la sole ou le turbot. En outre, les pêcheurs de Normandie sont familiers des variations de cette manne capricieuse et savent bien que son abondance ou sa rareté au moment de la " harengaison " dépendent en partie de facteurs d'ordre climatique $^{30}$. De ce point de vue, il est donc tentant d'y voir un argument de circonstance contre le chalut relevant de ce que Geneviève Delbos a proposé d'appeler une " rhétorique du dépeuplement ${ }^{31}$ ".

S'il est douteux que l'usage du chalut au large des côtes de HauteNormandie ait pu avoir un impact quelconque sur la ressource en hareng, il est possible en revanche qu'il ait occasionné l'épuisement ponctuel et localisé de certains fonds de pêche, dont les espèces sédentaires ont pu se trouver temporairement surexploitées. Les travaux menés dans le domaine de l'écologie historique depuis les années 2000 invitent en effet à accorder un certain crédit aux propos du maître Jacques Ville lorsqu'il explique, au cours de son interrogatoire, que " depuis quatre ans que cette pêche

28. Pavé, 2013, p. 173.

29. ADSM, 4/M/185, Lettre de l'écrivain des classes de Dieppe au commissaire général de la Marine, 28 novembre 1818, 9 heures du matin.

30. Smith, 1994, p. 8-34.

31. Delbos, 1989, p. 52-53. 
se fait dans les eaux du quartier de Dieppe, les produits de la pêche aux cordes ont diminué successivement, jusqu'au point de devenir nuls ${ }^{32}$ ". Il est intéressant de noter ici que des plaintes très semblables s'élèvent à la même époque de l'autre côté de la Manche à mesure que la pêche au chalut tend à se développer et à devenir hégémonique, au détriment d'une grande diversité de pratiques implantées localement ${ }^{33}$. Par ailleurs, même au sein d'une administration de la Marine fermement attachée au principe de la liberté de la pêche, certains s'accordent à reconnaître que l'usage du chalut a des effets bien réels sur la ressource. C'est le cas par exemple de l'officier Foubert, chargé en 1822 d'une longue enquête sur « les causes du désempoissonnement des côtes de Dieppe et de celles des environs ", qui considère que :

Ce filet [...] contribue à ce désempoissonnement parce que les côtes de Dieppe sont exploitées par un trop grand nombre de chalutiers proportionnellement à l'étendue des fonds sur lesquels ils exercent leur industrie. Le chalut prend beaucoup plus de poisson que tout autre filet relativement au nombre d'hommes qu'il emploie. [...] On ne doit pas s'étonner que cette grande destruction ait lieu plutôt dans les parages de Dieppe que partout ailleurs, puisque ce port par sa proximité de la capitale sera toujours fréquenté par une très grande quantité de pêcheurs de nos autres côtes. On doit aisément concevoir d'après cela que dès l'instant où le nombre de ceux du pays joint aux étrangers dépasse de certaines bornes, la reproduction du poisson n'est plus en rapport avec sa consommation ${ }^{34}$.

Partant de là, il pourrait être tentant d'interpréter les violences commises contre les chalutiers comme l'expression en acte d'une véritable " économie morale de la nature ", dont les principes dicteraient un bon usage des ressources tirées de la mer $^{35}$. Cette piste mériterait assurément d'être

32. ADSM, 4/M/185, Procès-verbal des interrogatoires des 30 novembre, $1^{\text {er }}$ et 2 décembre 1818. Parmi les travaux ayant documenté l'impact ancien des activités de pêche sur les écosystèmes côtiers, voir notamment. Jackson et al., 2001 ; Holm et al., 2010 ; Thurstan, Hawkins et Roberts, 2014.

33. Cornish, 1824 ; Guerrish, 2000.

34. SHDV, Mar., CC 578 , Rapport sur les causes du désempoissonnement des côtes de Dieppe et de celles des environs, 22 novembre 1822, p. 16-17.

35. Voir par exemple Cadigan, 1999, p. 12, qui propose une lecture écologique de la défense des coutumes locales régulant l'accès à certains territoires de pêches situés sur les côtes de Terre-Neuve. 
creusée, en se gardant toutefois du double écueil de l'idéalisation et de l'essentialisation des communautés de pêcheurs, dont l'attitude à l'égard du chalut est pour le moins ambivalente. On sait par exemple qu'à l'époque des faits rapportés ci-dessus, certains pêcheurs de Dieppe ont pris l'habitude de s'embarquer comme matelots à bord des barques de Honfleur et que plusieurs maîtres ont même commencé à instituer l'usage d'armer pour la pêche au chalut dans l'intervalle qui sépare la saison du hareng de celle du maquereau. Ainsi, lorsque le commissaire de police du port tente de verbaliser un chalutier du Pollet au mois de janvier 1817, un attroupement d'une centaine de personnes se crée aussitôt sur les quais pour s'opposer à la saisie de ses filets ${ }^{36}$. En d'autres termes, ce n'est pas tant le chalut en soi qui suscite l'opposition des acteurs de la communauté, que le chalut en tant qu'il est utilisé sur des fonds « dont la nature semble leur avoir dévolu exclusivement l'exploitation " par des "étrangers " qui ne reconnaissent et ne respectent pas leurs usages locaux ${ }^{37}$.

De fait, son emploi en toute saison par les pêcheurs « de la côte du Sud » va à l'encontre des « règles d'œconomie » qui encadrent la pratique de la pêche à Dieppe depuis le XVIII ${ }^{\mathrm{e}}$ siècle $^{38}$. Celles-ci consacrent en effet une organisation fondée sur la complémentarité entre les différentes pêches - ou " métiers » - qui se succèdent au cours de l'année, au gré d'un calendrier saisonnier dont l'établissement par les membres de la communauté vise à empêcher que la pratique des unes ne nuise à celle des autres ${ }^{39}$. Nullement liés par ces règles tacites suivies localement, les chalutiers de Honfleur sont ainsi accusés de faire concurrence aux équipages du lieu en apportant sur le marché des quantités importantes de poisson au moment même où les cargaisons de hareng frais doivent être écoulées rapidement. Par ailleurs, ils exploitent des fonds qui sont traditionnellement laissés au repos pendant l'automne afin que la ressource y soit plus abondante en hiver, une fois la " harengaison " terminée. Enfin, l'utilisation d'engins de pêche traînants

Pour une recension et une critique des recours au concept d' « économie morale " par les historiens du monde de la pêche : Payne, 2013.

36. ADSM, 4/M/185, Rapport du commissaire de police de Dieppe, 5 janvier 1817.

37. ADSM, 4/M/185, Lettre du commissaire de l'inscription maritime de Dieppe au commissaire de l'inscription maritime de Honfleur, 25 avril 1817.

38. Grancher, 2015, p. 420-423. Cette expression est tirée d'un "mémoire des intéressés à la pesche du harang " de Dieppe, daté du 31 décembre 1733 (Paris, Archives nationales, fonds Marine, $\mathrm{C}^{5} 32$, fo $82-85$ ).

39. Sur les concepts de saison et de métier dans le monde de la pêche, voir Jorion, 1979. 
comme le chalut est difficilement compatible avec celle d'engins dérivants comme les sennes qui servent à intercepter les bancs de harengs lors de leur passage à proximité des côtes normandes ${ }^{40}$. C'est la raison pour laquelle il existe à Dieppe, comme dans de nombreuses communautés de pêcheurs, des conventions tacites définissant ce que certains ethnologues ont proposé d'appeler des " technotopes ", à savoir la combinaison d'un lieu et d'une technique de pêche dans une période de temps donnée afin de capturer une espèce en particulier ${ }^{41}$. Cependant, les nombreux accidents qui surviennent sur les lieux de la pêche suggèrent que ces conventions locales ne sont guère respectées, de sorte que «les marins de Dieppe se plaignent de ce qu'ils ne peuvent plus mettre de filets à la mer, parce que « les bateaux chalutiers, ne respectant aucune limite, vont la nuit à travers tout, enlèvent $\&$ et détruisent leurs filets ${ }^{42}$ ".

À côté de ces griefs d'ordre écologique ou économique exprimés directement par les acteurs, soit au cours de l'émeute, soit au cours de l'enquête qui l'a suivie, ce conflit présente également une dimension sociale, dont la présence, parmi la foule réunie sur les quais le 28 novembre au matin, de femmes, d'enfants et "d'individus de toutes professions » est toutefois l'un des seuls indices fournis par les sources relatives à cet événement ${ }^{43}$. D'un coût relativement modique, le chalut est un engin de pêche qui n'implique pas, à l'inverse de ceux en usage à Dieppe, de recourir au système de l'armement à la part, c'est-à-dire à la fourniture en commun par le maître, les matelots et l'armateur des pièces de cordes ou de filets nécessaires pour former un engin de pêche complet - ce qu'on appelle une "tessure " ${ }^{44}$. Or ce système permet l'implication de nombreux acteurs extérieurs aux sociétés d'armement, notamment des anciens, des infirmes ou des veuves, qui tirent un revenu du laçage à domicile des filets en les revendant à des matelots qui en sont eux-mêmes dépourvus (car ils ne sont pas mariés et ne peuvent donc les faire fabriquer par leur femme et leurs enfants),

40. Sur les conflits d'usage entre techniques de pêche, voir Dufour, 1987.

41. Fay, 1993 ; Bouju, 1995.

42. ADSM, 4/M/185, Lettre du maire de Dieppe au sous-préfet de Dieppe, 28 novembre 1818.

43. ADSM, 4/M/185, Lettre l'écrivain des classes de Dieppe au commissaire général de la Marine, 28 novembre 1818, 9 heures du matin. La lecture proposée dans ce paragraphe suivant s'appuie très largement sur Dardel, 1941, p. 214-216.

44. Parmi l'abondante bibliographie relative à l'armement à la part, ou share system, on peut notamment se reporter à Acheson, 1981 ; Bidet, 1974 ; Breton, 1981 ; van Ginkel, 2009, p. 170-183 ; Howard, 2012. 
ou en les "faisant pêcher " par un équipage en échange d'une part sur le produit de la vente du poisson. À l'inverse, la diffusion du chalut est synonyme d'individualisme halieutique puisqu' elle permet aux armateurs, ainsi qu'à quelques maîtres de bateau aisés, de devenir les uniques détenteurs des engins de pêche, entraînant au passage les matelots dans la condition salariale ${ }^{45}$.

Pour toutes ces raisons, il est tentant, finalement, de regarder cet événement comme une émeute à caractère luddite. Mais est-ce là, toute proportion gardée, une grille de lecture opérante, ou du moins intéressante ? Si aucun cas de bris de chalut n'a été rapporté par les autorités locales, il faut tout de même noter que dans l'après-midi du 27 novembre, " les femmes du Pollet menaçaient de mettre le feu aux barques pendant la

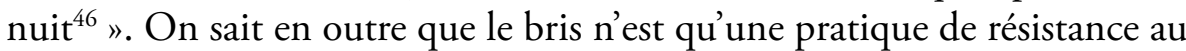
changement technique parmi d'autres et qu'elle prend sens au sein d'une pluralité de modes d'action allant de la pétition à l'émeute en passant par le recours judiciaire, dont la finalité est d'abord la défense d'un mode de vie mis en péril par l'adoption d'une nouvelle machine ${ }^{47}$. À cet égard, les violences perpétrées à l'encontre des chalutiers de Honfleur ne sont donc pas sans faire écho aux conflits sociotechniques qui accompagnent l'essor de la mécanisation de part et d'autre de la Manche : en bloquant l'accès au marché local du poisson pour les pêcheurs étrangers à la communauté, la population maritime dieppoise tente en effet d'instaurer un rapport de force pour négocier les usages d'une technique qui menace de détruire jusqu'à ses ressources, ses coutumes et même ses propres outils.

\section{Bibliographie}

AваD Reynald, Le Grand marché. L'approvisionnement alimentaire de Paris sous l'Ancien Régime, Fayard, Paris, 2002.

ACERra Martine et Zysberg André, L'Essor des marines de guerres européennes (vers 1680-vers 1790), SEDES, Paris, 1997.

Acheson James M. , «Anthropology of fishing », Annual Review of Anthropology, vol. 10,1981 , p. $278-280$.

45. Sur les conséquences sociales de la diffusion du chalut, voir le bel article de Rule, 1976.

46. ADSM, 4/M/185, Lettre de l'écrivain des classes de Dieppe au commissaire général de la Marine, 29 novembre 1818 , au soir.

47. Jarrige, 2009, p. 145-195. 
Armiero Marco, "La risorsa contesta: norme, conflitti e technologie tra i pescatori meridionali (xIX sec.) ", Meridiana, n 31, 1998, p. 179-206.

Bidet Jacques, "Sur les raisons d'être de l'idéologie : les rapports sociaux dans le secteur de la pêche ", La Pensée, no 174, 1974, p. 53-67.

Bouju Stéphane, "Anthropologie et halieutique : réflexion sur l'élaboration d'une typologie et sur l'intérêt de l'utilisation de la notion de technotope ", dans Francis Lalö̈, Hélène Rey et Jean-Louis Durand (dir.), Questions sur la dynamique de l'exploitation halieutique, ORSTOM, Paris, 1995, p. 245-262.

Bourdeau Vincent, Jarrige François et Vincent Julien, Les Luddites. Bris de machines, économie politique et histoire, Éditions ère, Maison-Alfort, 2006.

Breton Yvan, "L'anthropologie sociale et les sociétés de pêcheurs. Réflexions sur la naissance d'un sous-champ disciplinaire ", Anthropologie et Sociétés, vol. 5, $\mathrm{n}^{\circ} 1,1981$, p. 7-27.

Cabantous Alain, Les Citoyens du large : les identités maritimes en France (XVII XIX siècle), Aubier, Paris, 1995.

Cadigan Sean, "The Moral Economy of the Commons: Ecology and Equity in the Newfoundland Cod Fishery, 1815-1855 », Labour/Le Travail, vol. 43, 1999, p. 9-42.

CAPtiER Jacques, Étude historique et économique sur l'inscription maritime, V. Giard \& E. Brière, Paris, 1907.

Connish James, A view of the present State of the Salmon and Channel Fisheries, and of the Statute Laws by which they are regulated; showing, that it is to the defects of the latter that the present scarcity of the fish is to be attributed, Londres, 1824.

DARDEL Éric, La Pêche harenguière en France : étude d'histoire économique et sociale, PUF, Paris, 1941.

Delbos Geneviève , "De la Nature des uns et des autres. À propos du dépeuplement des eaux ", dans Nicole Mathieu et Marcel Jollivet (dir.), Du rural à l'environnement : la question de la nature aujourd'hui, L'Harmattan, Paris, 1989, p. 50-63.

Dufour Annie-Hélène, "Poser, traîner : deux façons de concevoir la pêche et l'espace ", Bulletin d'Ecologie humaine, vol. 5, n 1, 1987, p. 23-45.

FAget Daniel, Marseille et la mer. Hommes et environnement marin (XVIIIXX siècle), PUR, Rennes, 2011.

Farge Arlette et Revel Jacques, Logiques de la foule, Hachette, Paris, 1988.

FAY Claude, "Repères technologiques et repères d'identités chez les pêcheurs du Macina (Mali), dans Marie-José Solivet et Diana Rey-Hulman (dir.), Jeux d'identités. Études comparatives à partir de la Carä̈be, L'Harmattan, Paris, 1993, p. 167-202. 
Fourt Maïa, Faget Daniel et Pérez Thierry, "Fighting the Minotaur: Resistance to technological change in the Mediterranean sponge fishing industry, 1840-1922 ", International Journal of Maritime History, vol. 32, n 2, 2020, p. 337-353.

Gersuny Carl et Poggie John J., "Luddites and Fishermen. A Note on Response to Technological Change ", Maritime Studies and Management, vol. 2, 1974, p. 38-47.

Gordon H. Scott, "The trawler question in the United Kingdom and Canada ", Dalhousie Review, 1951, p. 117-127.

GrANCHER Romain, "Écrire au pouvoir pour participer au gouvernement des ressources. L'usage des mémoires dans la controverse sur le chalut (Normandie, premier XIX siècle) ", L'Atelier du CRH, 13, 2015, p. 175-212, https://journals. openedition.org/acrh/6560.

Grancher Romain, "Les usages de la mer. Droit, travail et ressources dans le monde de la pêche (Dieppe, années 1720-années 1820) ", thèse de doctorat d'histoire, Université de Rouen, 2015.

Guerrish Margaret, «Following the Fish: Nineteenth-Century Migration and the Diffusion of Trawling ", dans David Starkey, Chris Reid et Neil Ashcroft (dir.), England's sea fisheries, Chatham, Londres, 2000, p. 112-118.

Hoвsваwm Eric J. et Rudé George, Captain Swing, Penguin University Books, Londres, 1973.

Holm Poul et al., " Marine animal populations: A new look back in time", dans Alasdair McIntyre (dir.), Life in the world's oceans. Diversity, Distribution and Abundance, Blackwell, Chichester, 2010, p. 3-23.

Howard Penny M., "Sharing or Appropriation? Share System, Class and Commodity Relations in Scottish Fisheries ", Journal of Agrarian Change, vol. 12, $\mathrm{n}^{\circ} 2$, 2012, p. 316-343.

JaCKson Jeremy B. C. et al., "Historical Overfishing and the Recent Collapse of Coastal Ecosystems ", Science, vol. 293, n 5530, 2001, p. 629-638.

JARRIGe François, Au temps des "tueuses de bras". Les bris de machines à l'aube de l'ère industrielle (1780-1860), PUR, Rennes, 2009.

Jones Peter, "The long 'lost' history of bottom trawling in England, c. 13501650 ", International Journal of Maritime History, vol. 30, n 2, 2018, p. 201-217.

JoRION Paul, "Les deux concepts fondamentaux de la pêche artisanale. La « saison " et le " métier " à Houat (Morbihan) ", Ethnologie française, vol. 9, n 2 , 1979, p. 135-146.

McCaY Bonnie J., "The Culture of the Commoners. Historical observations on old and new world fisheries", dans James M. Acheson et Bonnie J. McCaY, 
The Question of the Commons. The culture and ecology of communal resources, The University of Arizona Press,Tucson, 1987, p. 195-216.

Mollat Michel (dir.), Histoire des pêches maritimes en France, Éditions Privat, Toulouse, 1987.

PavÉ Marc, La Pêche côtière en France (1715-1850). Approche sociale et environnementale, L'Harmattan, Paris, 2013.

Payne Brian, «Local Economic Stewards: The Historiography of the Fishermen's Role in Resource Conservation ", Environmental History, vol. 18, 2013, p. 29-43.

Roberts Callum M., The Unnatural History of the Sea, Island Press, Washington, 2007.

Robinson Robb, The Rise and Fall of the British Trawl Fishery, University of Exeter Press, Exeter, 1996.

RulE John, "The smacksmen of the North Sea. Labour recruitment and exploitation in British deep-sea fishing, 1850-1890 ", International Review of Social History, vol. 21, n 3, 1976, p. 383-411.

SMith Tim D., Scaling Fisheries: The Science of Measuring the Effects of Fishing, 1855-1955, Cambridge University Press, Cambridge, 1994.

Subramanian Ajantha, "Modernity from below: local citizenship on the south Indian coast ", International Social Science Journal, vol. 155, n 175,2003 , p. 135-144.

Thompson Edward P., "L'économie morale de la foule anglaise au XVIII ${ }^{\mathrm{e}}$ siècle " (1971), dans Idem, Les Usages de la coutume: tradition et résistances populaires en Angleterre, XVII $-X I X^{e}$ siècle, traduit de l'anglais par J. Boutier et A. Virmani, EHESS/Gallimard/Seuil, Paris, 2015 [1991], p. 251-329.

Thompson Edward P., La Formation de la classe ouvrière anglaise, Gallimard : le Seuil, Paris, 1988 [1963].

Thurstan Ruth H., Hawkins Julie P. et Roberts Callum M., « Origins of the bottom trawling controversy in the British Isles: $19^{\text {th }}$ century witness testimonies reveal evidence of early fishery declines ", Fish and Fisheries, vol. 15, 2014, p. 506-522.

UeRTEAga Luis, La tierra esquilmada. Las ideas sobre la conservación de la naturaleza en la cultura española del siglo XVIII, CSIC, Madrid, 1987.

VAlin René-Josué, Nouveau commentaire sur l'ordonnance de la Marine du mois d'Août 1681, La Rochelle, 1760, 2 tomes.

van Ginkel Rob, Braving Troubled Waters. Sea Change in a Dutch Fishing Community, Amsterdam University Press, Amsterdam, 2009 
La guerre du chalut

\section{L'auteur}

Romain Grancher est chargé de recherche au CNRS, membre de l'unité TEMOS UMR 9016. Il travaille à une anthropologie historique et de l'histoire des savoirs et des techniques. Contact : romain.grancher@univ-ubs.fr 